\title{
Modeling A Wireless Network For International Space Station
}

\author{
Richard Alena \\ NASA Ames Research Center \\ MS 269-4, Moffett Field, CA 94035 \\ calena@mailarc.nasa.gov \\ 650-604-0262 \\ Ece Yaprak \\ College of Engineering \\ Wayne State University \\ $48554^{\text {th }}$ Street, Detroit, MI 48202 \\ yaprak@enr.wayne.edu \\ Saad Lamouri \\ CACI Products Company \\ 3333 N. Torrey Pines Ct, La Jolla, CA 92037 \\ saad@modsim.com
}

Abstract - This paper describes the application of wireless local area network (LAN) simulation modeling methods to the hybrid LAN architecture designed for supporting crew-computing tools aboard the International Space Station (ISS). These crew-computing tools, such as wearable computers and portable advisory systems, will provide crew members with real-time vehicle and payload status information and access to digital technical and scientific libraries, significantly enhancing human capabilities in space. A wireless network, therefore, will provide wearable computers and remote instruments with the high performance computational power needed by next-generation "intelligent" software applications: Wireless network performance in such simulated environments is characterized by the sustainable throughput of data under different tiaffic conditions. This data will be used to help plan the addition of more access points supporting new modules and more nodes for increased network capacity as the ISS grows.

\section{Table Of Contents}

1. Introduction

2. Network Architecture On The ISS

3. Wireless Network Simulation $\therefore$

4. Conclusion.

\section{Introduction}

NASA Ames Research Center's Intelligent Mobile Technologies (IMT) project is developing and implementing information technologies to enhance the International Space Station (ISS) computational capabilities. The ISS will be the largest and most complex spacecraft ever operated with sophisticated space-based laboratories incorporating hundreds of experiments ranging from life sciences to structural mechanics. These experiments will be largely automated to minimize crew workload. To take advantage of the operational adaptability of the human element, the crew needs ready access to mission data. The current limitations of the on-board data system make it difficult for the crew to access avionics data. A mobile intranet based on open-system standards for software development, and data communications and networking can efficiently provide a universal monitoring interface.

Portable Advisory Systems will provide unique capabilities for human interaction with data systems by focusing on next-generation hand-held and wearable computing devices that are closely coupled with ground facilities such as mission and payload control centers, science laboratories and universities allowing complex collaborative interactions that will rely on industrystandard data interchange protocols, computing resources and end-user applications. This will extend the on-board flight vehicle computing environment into cyberspace, allowing virtual yet secure interactions between flight crew members, ground controllers and principal investigators. Mobile handheld computers will allow a crew member to communicate with another crew member; monitor vehicle and payload status, and receive ground communications at any location while floating in a Station module, without any physical power or data connection to hinder movement or action. Wearable computing devices allow a crew member to perform vehicle maintenance activities and field exploration using techniques that do not interfere with vision or manual dexterity. 
The primary benefit of mobile computing is improving human-computer interaction. Crew support applications for mobile computers help crew members accomplish their assigned tasks by acting as data display and manual input devices for onboard data systems. They could be used for note taking, messaging, activity scheduling, decision support, reference material access, on-board training, and recreational uses. ${ }^{i}$ A distributed network architecture of notebook computers or pen-based hand-held data terminals, communicating through wireless networks to dedicated data servers are proposed for these applications. The on-board data systems would support these mobile devices by acting as file and communications servers for client applications. It is in this area where the full benefits of mobile computing technology can be greatest by increasing the efficiency of crew operations.

Another benefit of mobile computing is the ability to access data from a variety of physical locations. This is the advantage provided by distributed computing. A given crew member could perform a wider range of tasks if they could be performed while mobile.

The ISS consists of a series of modules connected by docking nodes that house laboratories and habitats. Each module contains a series of payload racks that contain experiments and the data systems needed to operate the experiment and collect the data. Additionally, the avionics system allows the Station to be reboosted into a higher orbit and controls the generation of power and life support. A data system for supporting vehicle and payload operations would have to allow connections to this wide variety of data sources. The network is the logical choice for this and the use of Internet protocols allows rapid development and deployment of custom application software. Some advantages of wireless network technology for the Space Station are:

- Wireless networks of mobile computers can be used aboard the ISS to enhance payload operations, allowing crew members to access payload status remotely, thereby supporting multiple payloads simultaneously.

- A wireless network can be upgraded by simply bringing up new components such as hand-held computers and servers.

- The wireless network can support remote sensors for microgravity and other environmental conditions that could be placed anywhere within the habitable volume.

- The network could support crew functions such as communication, inventory control and computer-based recreation.
- An immediate savings in cable weight and installation cost is realized using this technology.

\section{Wireless Network Architecture On The ISS}

Wireless data links can be organized into networks that provide connectivity of computer system resources between multiple users and devices. The evolution of wireless networking is based on current wired network methods such as the Ethernet and the Token Ring using extensions to existing network protocols. Current wireless products use either spread-spectrum radio or infra-red optical methods for data communication. They are available in a variety of interface formats that allow operation of the wireless data links with wired networks, resulting in a hybrid networking system.

The payload support computing infrastructure aboard the ISS consists of a distributed system of servers and mobile client computers based on current laptop technology. These components are connected through wired and wireless segments of the hybrid Operational LAN. The components of a wireless LAN consist of a wireless network interface card (NIC) and a wireless local bridge (access point) to the wired segment. Most wireless NICs interface computers to the wireless network through the access point by implementing a carrier sense multiple access with collision avoidance (CSMA/CA) protocol, and modulating the data signal with a spreading sequence. The IEEE 802.11 standard defines the access protocol and modulation methods.

Networks are often characterized by the area of coverage. A wide area network (WAN) uses longrange radio and telephone cables to maintain coverage over a city, state or country. A LAN usually covers a building or suite of rooms. Many LAN segments can operate together to cover a larger number of users, with concurrent network management and throughput advantages. A new type of network, the personal area network (PAN), uses wireless methods for limited coverage. PANs cover a smaller area than a LAN, typically only a room, and generally allow dynamic entry and exit of nodes from the network. Two types of architecture can be used for wireless PANs, the peerto-peer model and the client/server model.

The peer-to-peer model allows computer device nodes with fairly equal capabilities to interchange data with each other. Each node incorporates both client and server functions. There is no central node mediating the traffic or managing the network. A collection of nodes can setup an "ad-hoc" network by using the same type of asynchronous network access used by the Ethernet. For example, a peer-to-peer PAN would allow distribution of attendee contact information and 
presentation slides to any perșon with a mobile computer node who enters the meeting room. The presenter's computer would recognize the new participant in the PAN, and send the data to him if desired.

The client/server model allows connecting a wireless PAN to a wired LAN using a node designated as the "access point" to the wired LAN. The access point computer node must be capable of communicating over both the wired and wireless data links, acting as a network server. The access point allows a wireless client node to access computing resources and data on the entire LAN, significantly extending functionality.
Clusters of peer-to-peer nodes could coexist within this client/server configuration resulting in a hybrid architecture.

Multiple access points can be used to configure a "micro cellular" wireless architecture such as that shown in Figure 1. Access points integrate wireless 'PANs with wired Ethernet LANs by filtering packets from the LAN and forwarding the appropriate ones to the wireless PAN. The access point also listens to the wireless PAN and forwards appropriate traffic to the LAN. If a wireless node is unreachable, the access point can store the data and forward it to another access point.

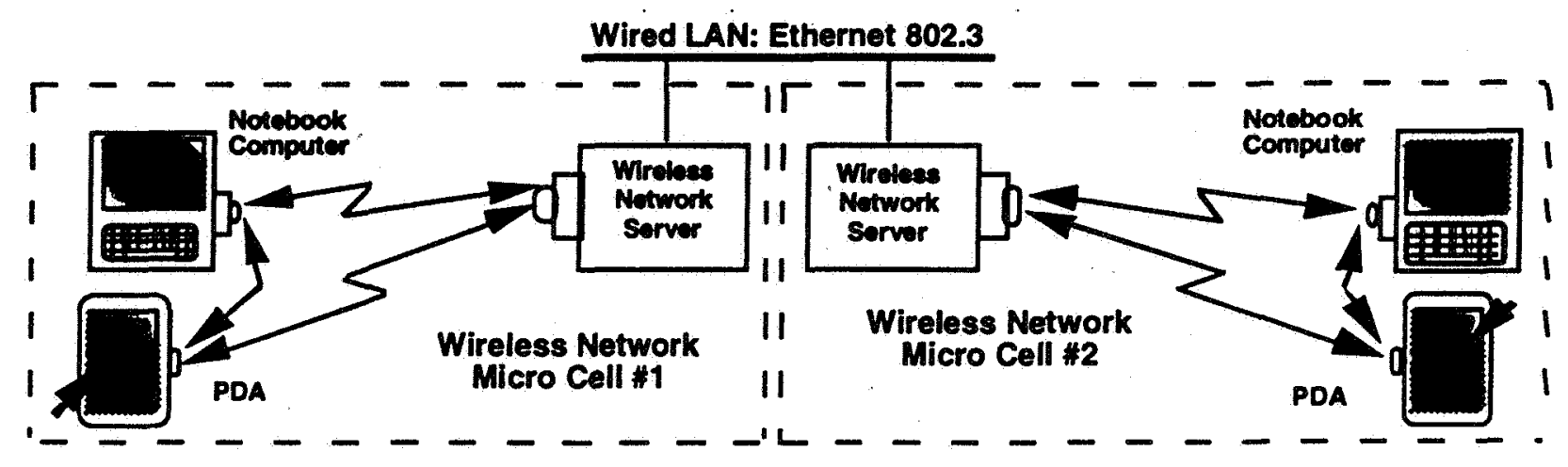

Figure 1. Wireless Network Micro Cellular Architecture

Significant differences exist between wireless media and wired LANs: the bit error rate for wireless transmission is much higher than wired media, and a wireless transceiver is unable to determine if another. node is transmitting since its own signal swamps out any remote transmission. Also the network configuration is dynamic, with nodes entering and leaving the PAN at any time.

The Wireless Network Experiment (WNE) was one of the Experiments conducted by the Shuttle Mir Science Program to evaluate advanced technology for use on the International Space Station in 1996 which measured wireless network throughput as a handheld computer was moved from one location to the other.

The WNE consisted of three computers. Three nodes allowed measurement of network throughput while two nodes were simultarieously. sending and receiving data from the wireless network server (WNS). This configuration simulates a real computer network by forcing arbitration between nodes for network access. The experiment varied the distance between the three: nodes while compiling real-time throughput data graphs for each physical location. The network radios were made by Proxim Corporation and are products that helped to define the 802.11 wireless network standard.
A fundamental characteristic of wireless networks is that a given node can only "see" traffic specifically addressed to it. Therefore, to measure wireless network traffic accurately, a single node had to handle all the network traffic and simultaneously monitor it. This required use of the high-performance WNS workstation to store the data to be transferred on the network and also run the network monitor program. The variations of network throughput as a function of distance between nodes, and in the presence of various shielding structures and electromagnetic interference were measured both on the ground and in the spacecraft.

\section{Wireless Network Simulation}

Network simulation provides a method of modeling a proposed network to determine its performance characteristics, conducting what-if-analyses, and planning for increased network capacity. In this paper, a network simulation package, COMNET III from CACI Products Company, was used [4] to model the operation of the OpsLAN aboard the ISS. This was done to provide information regarding network throughput limitations as the OpsLAN is expanded to multiple 
cells. The (CONNE: I smblation fost incorporatcs an IEEE 802.11 model that allows accurate defermination of throughput for network configurations proponed for the ISS. Determining the bottlenecks fir a given configuration allows recontiguring the network to produce better overall throughput. The ISS LAN environment will consist of multiple wireless cells using access points to connect to wired segments and servers. A wireless backbone may solve the problems of interconnecting the cells between modules. All these network elements can be modeled prior to deployment resulting in optimal network design.

A number of different hybrid wireless network configurations were modeled and compared. These included a 2- and 4-node wircless (11:1:2:802.11) links with a i-node Fthernet (11:1:1: 802.1$)$ connection. The simulations were pertormed for different levels of traffic, including underutilized and saturated, to cvaluate network performance under different comdilions. In Fig. 2. a 4-node wireless with a 3-node - ethernet is modeled and compared with a 2-node wireless with a 3-node ethernet.

Different traffic conditions and tile sizes (as seen in Fig. 3.) were simulated and reports were generated describing the performance of the modeled networks. Link utilization, and latency were some of the performance characteristics studied.

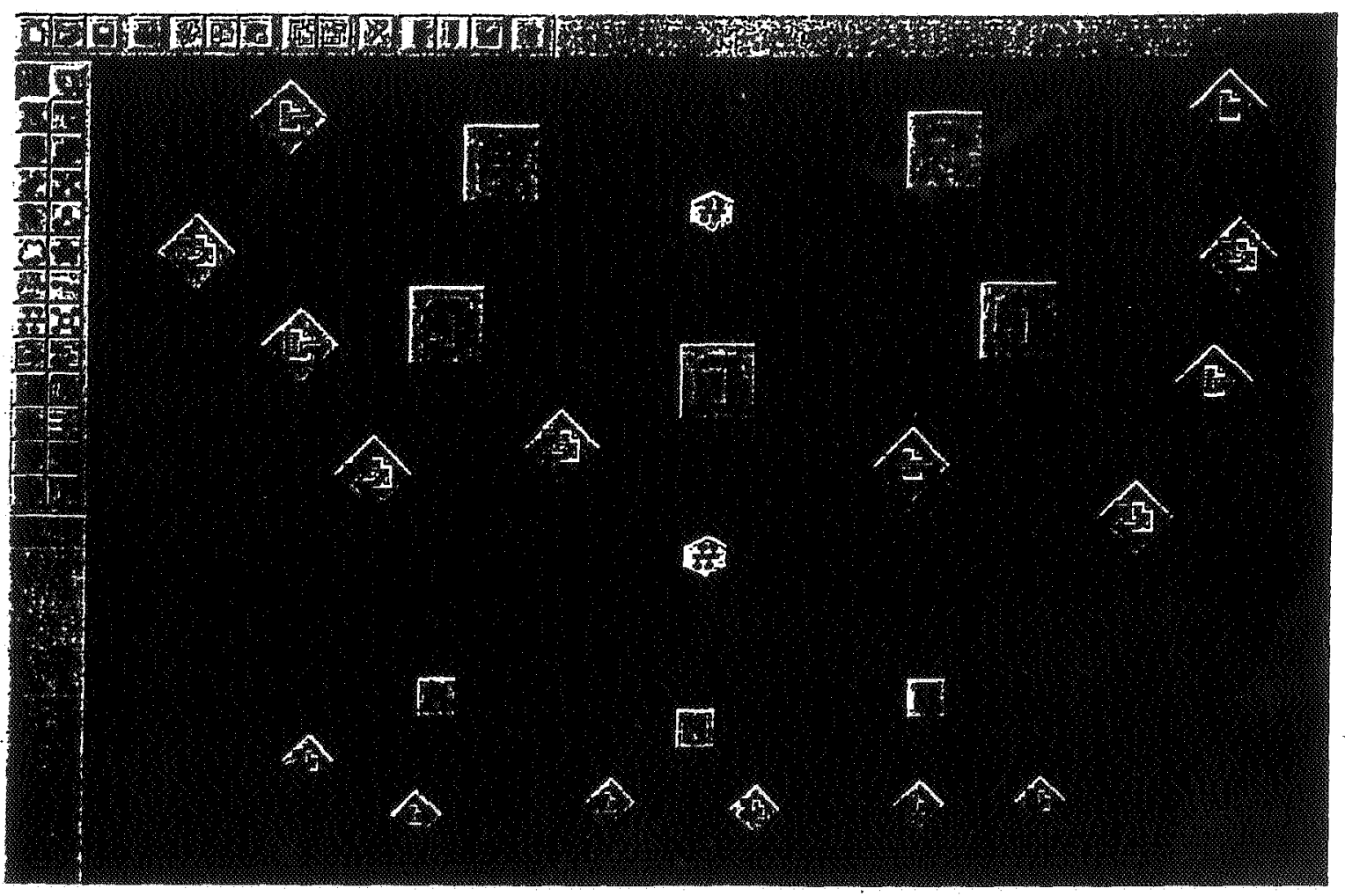

Fig.2. Wireless and ethernet simulation model.

Fig. 4 shows link utilization in terms of different traffic loading conditions for 2- and 4-wireless node models. ror $2 \mathrm{~K}$ and $20 \mathrm{~K}$ message sizes, both models were underutilized. For the wireless 4 -node model, link utilization declined as the link was saturated due to increased collisions. This data provides a new benchmark on which new simulations can be conducted to address the future communication scenarios on the ISS. 


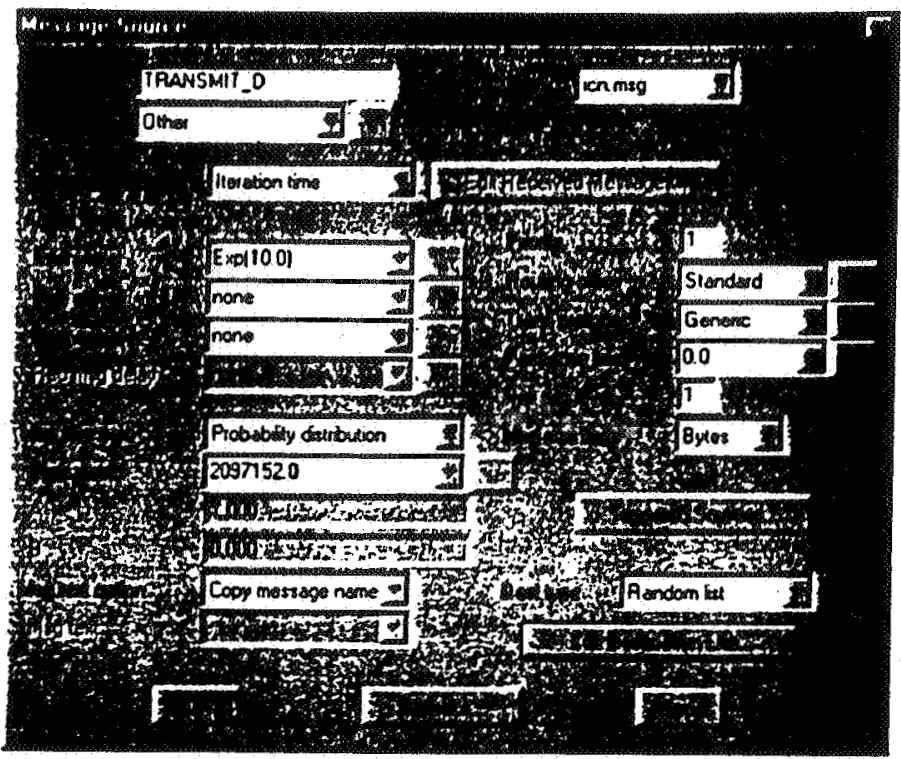

Fig.3. Traffic generation.

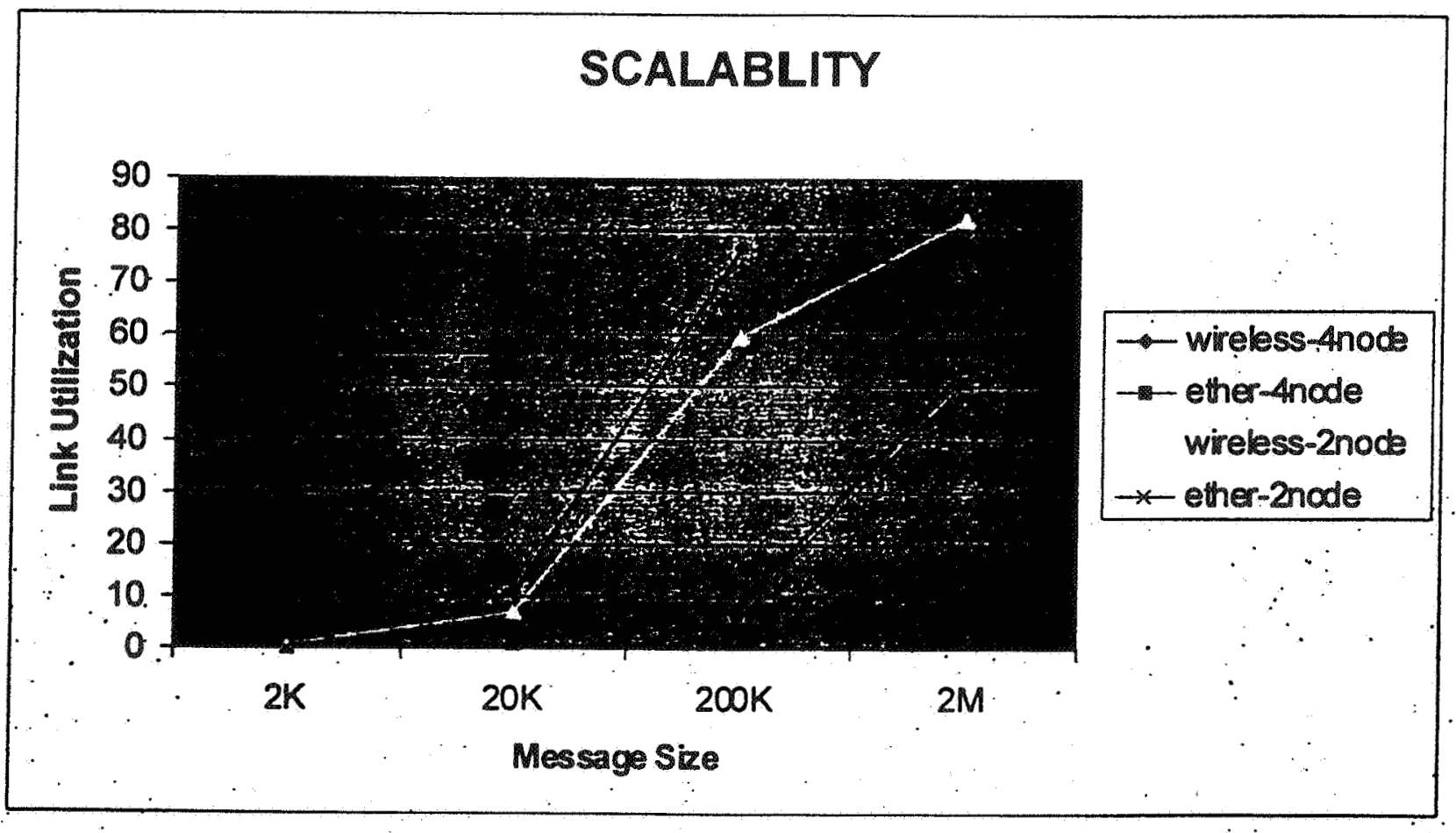

Fig. 4. Link utilization in terms of message size for 2 and 4-wireless-node models.

\section{Conclusion}

A wireless LAN simulation model was developed. Our studies showed that for large file sizes, the link utilization between nodes, connected through a wireless network, decreases due to collisions while the communication between wireless nodes and ethernet node is not saturated. Results obtained from our simulation runs were similar to those obtained from the wireless network experiment, which was conducted by the Shuttle Mir Science Program, thereby attesting to the reliability and validity of our simulation resulis. 
Future work should concentrate on multi-cell wireless systems connected through Ethernet systems.

\section{Acknowledgement}

Authors would like to acknowledge CACI Products Company, of La Jolla, California for their generous permission to use the software.

\section{References}

[1] J. Geier, Wireless LANs - Implementing Interoperable Networks, Macmillan Network Architecture Development Series, 1999.

[2] T. W. Madron, LANs - Applications of IEEE/ANSI 802 Standards, John Wiley \& Sons, Inc., 1989.

[3] P. T. Davis, C. R. McGuffin, Wireless Local Area Networks - Technology, Issues, and Strategies, MoGrawHill, 1995.

[4] COMNET III Reference Guide, CACI Products Company, 1999.

Richard Alena received his Master of Science degree in Electrical Engineering and Computer Science from the University of California at Berkeley in 1980. Currently working as a.computer scientist and engineer at NASA Ames Research Center, he previously developed hightech products such as multiprocessor computer systems, implantable biosensors and computed tomography medical scanners for industry. Rick now heads the. Intelligent Mobile Technology project team developing innovative concepts and devices that enable people to interact with computers, instruments and spacecraf in more effective and complex ways. In 1995, he was the chief engineer of the Wireless Network Experiment that flew a radio nerwork aboard the Shuttle and Mir space station (see http://ic.arc.nasa.gov/ic/projects/WNE/). For the Haughton-Mars Science Expedition he has deployed the Mobile Exploration System in the Arctic "field environment, studying how digital technology can extend current capabilities for observation of geological and biological features. (see http://arcticmars.org)

Ece Yaprak received her BS in Electrical Engineering in 1980 from the University of Michigan-Dearborn, MS in Computer Engineering in 1984 and Ph.D. in 1989 from Wayne State University. Dr. Yaprak joined the Wayne State University in August 1993. Her specialty areas are Computer Networking and Communications. Prior to joining WSU, she worked for Western Michigan University, General Electric, Ford Motor Company. During the last seven years she has held faculty fellowships at NASA Lewis Research Center, Jet Propulsion Laboratory of California Institute of Technology, NAVY/ASEE Summer Research Faculty Fellowship at the U.S. NAVY Space and Warfare Systems Center (SPAWAR) and NASA Ames Research Center of Stanford University.

Saad Lamouri works as a modeling and simulation consultant at CACI Products Company in La Jolla, California. He received $a B S$ and $M S$ in electrical engineering from San Diego State University. He may be reached at saad@modsim.com. 